\title{
Optimized Cardiovascular Disease Detection and Features Extraction Algorithms from ECG Data
}

\author{
Sanjay Ghodake ${ }^{1}$ \\ MIT Academy of Engineering \\ Alandi, Pune, India
}

\author{
Shashikant Ghumbre ${ }^{2}$ \\ Govt. College of Engineering and \\ Research, Avasari, Pune, India
}

\author{
Sachin Deshmukh ${ }^{3}$ \\ Dr.BAMU, Dept. of CSIT \\ Aurangabad, India
}

\begin{abstract}
A heart disease called cardiovascular diseases (CVD) is another leading cause for the death. There are several reasons that lead the CVD in human beings. The early detect of CVD helps to take necessary medical attentions to prevent the harms. The conventional techniques for CVD detection were manual and expensive which often delivers the inaccurate diagnosis. Since from the last decade the other inexpensive Computer Aided Diagnosis (CAD) based methods gained significant medical attentions. The CAD based techniques mainly based on raw Electro Cardiogram (ECG) signals of patient for the accurate and economical detection of CVD at early stage. In recent past, there are several CAD systems designed for CVD diagnosis utilizing raw ECG signals, however accuracy of CVD detection utilizing ECG bothered through several issues of research like QRS beats extraction, artefacts, efficient features extraction. This research paper present CVD novel framework, utilizing raw ECG signals and designed hybrid pre-processing algorithm for extracting artefacts and noise through raw ECG signal. Further designed simple and efficient dynamic thresholding based technique to extract the beats such as $Q, R, S$, and ST segment through pre-processing ECG signal. Third step perform the fusion of extracted beats and apply the feature extraction method called Normalized Higher Order Statistic (NHOS). The normalized HOS techniques asses the complexity among all the QRS based beats and delivers the more unique features for the accuracy enhancement. The final step is the classification by using five different classifiers for the CVD detection. The simulation results presented in this paper demonstrate that proposed framework achieved the significant accuracy improvement.
\end{abstract}

Keywords-Electrocardiogram; heart disease; cardiovascular disease; hybrid filtering; features extraction; QRS and ST beats

\section{INTRODUCTION}

The electrical improvement of human heart is assessed by utilizing the different body habitats, which are appropriate, masterminded over the body. From the estimation results, paper can shape a thought of heart limit called as electrocardiogram (ECG) [1]. The ECG is utilized to analyze of coronary illness these days in clinical frameworks. The heart banner winds up critical biomedical signs. A contraption called ECG banner is utilized to see heart rhythms. In heart muscles, starting and spread of electrical potential is recorded in ECG banner. Depolarization of chamber is tended to by ECG banner and ventricle in one pattern of each heartbeat [2, 3]. ECG banner is more essential than other natural signs and it has specific morphological properties. This morphology apparently is familiar along examining different cardiovascular maladies. Banner preparing is familiar along investigating and assessment of ECG signals. The portrayal of ECG banner is six apexes and valleys. The key attributes of human ECG signal are appears in Fig. 1. As seen in Fig. 1, the key waves called P, Q, S, R, \& T and so on comprises the significant fragments of ECG signal called QRS complex, ST section, and PR interim [4,5]. The ECG signal has been assorted and comprehend the improvement of exactness estimation and reproducibility. ECG signal is troublesome while examination the sign because of undermined by clamour during obtaining. One of the most testing ECG signal is the extraction of clinical parameters to frame uproarious biomedical signs.

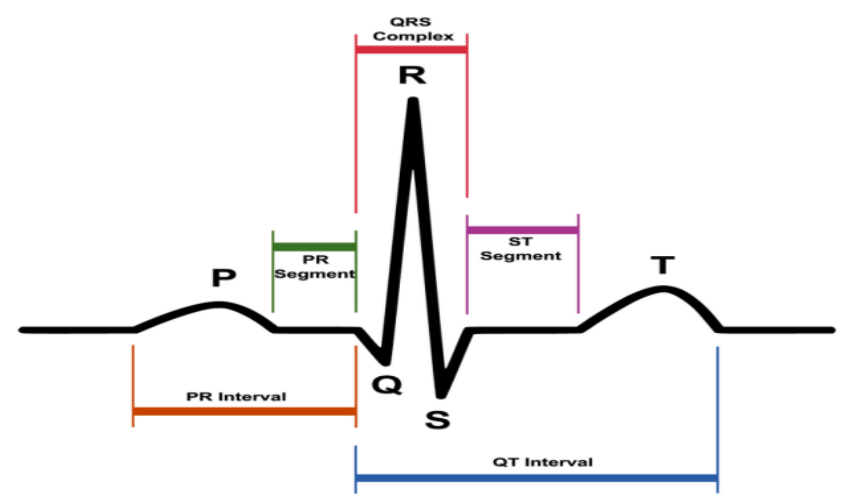

Fig. 1. Characteristics of ECG Signal [5].

By added substance high recurrence commotion, muscle relics or movement and standard meander cover signal in time and recurrence ECG signal is tainted. The issues are antiquities, which happen like an undesirable recurrence in signals. Simple channels can assist along taking care of these issues, yet they can cause nonlinear stage shifts, signal twisting. Computerized channels are progressively precise. The ECG signs can be harmed by various sorts of commotion. The way is required to have the pre-processing strategies to denoise the information ECG signal for the precise examination and finding utilizing the CAD frameworks. There are number of sifting strategies intended to denoise the ECG signals since from a decade ago detailed in [6]-[12], anyway the key worries for ECG signal processing is the multifaceted nature and productivity.

After the pre-processing of ECG signal, the following test is identified along the extraction of five pinnacles, for example, P, Q, R, S, \& T from the ECG signal. The Individual pulses in ECG information are basically described by such 
five pinnacles and valleys, namely, P, Q, R, S, \& T, and these can be utilized to recognize atypical heartbeat in CAD frameworks. For CVD location, the QRS complex and ST portion extraction assumes the huge job, as the both QRS complex and ST fragment contains the key data identified along heart conduct. Accordingly, the dynamic advance in CVD discovery primarily embraces the strategies to extricate the genuine QRS complex area. Various calculations dependent on subordinate [13], computerized channels [14], and wavelet change [15] have as often as possible been utilized for QRS recognition. Along the improvement of equipment condition, considerably more strategies receive wavelet changes. In wavelet-based procedures, the effectiveness of wavelet change emphatically relies upon the decision of the mother wavelets. Other discovery calculations proposed in the literary works including numerical morphology [16], shrouded Markov model [17], S-change [18], standard language structure [19], quadratic channel [20], multiresolution entropy [21], meagre representation [22], and particular worth deterioration (SVD) [23]. Key concern for QRS a complicated and ST segment extraction method has been complexity and accuracy while utilizing along machine learning techniques. The QRS complex and ST segments holds the big and complex data, which may take longer time for the prediction and may leads to inaccurate detection as well. Hence the QRS complex and ST segments needs to optimized further by utilizing the features extraction and normalization techniques before applying the machine learning methods for the early CVD detection.

Under this research, first described the hybrid filtering approach based on notch filtering and high-pass band filtering method for the pre-processing of raw ECG signal. After that designed simple and efficient algorithm for Q, R, S, \&T waves extraction and formation of QRS complex and ST segments. The QRS complex and ST segments further exploited for the NHOS based features extraction to boost the accuracy performance and robustness. The features extraction for training ECG dataset is further investigated utilizing five different machine learning based methods. In Section II, the review of recent filtering and CVD detection techniques presented. In Section III, the proposed methodology described. In Section IV, the result analysis, in Section V discussion. Finally in Section VI, conclusion and future work presented.

\section{RELATED WORK}

The CVD detection is challenging research problems due to the artefacts, baseline wander, noises, efficient extraction of QRS complex and ST segments, features construction and classification phases. This section describes review of current ECG based methods.

In [8], creator proposed the SVD approach for ECG denoising. The ECG signal is pulled in additional by white Gaussian clamour. ECG signal is deteriorating into symmetrical subspaces utilizing high choice estimation range instruments are done in this methodology. The commotion parts contained inside ECG signal that subspaces related to symmetrical change inside particular worth disintegration. SVD disposes of the subspace of clamour and the unfortunate sign parts.
In [9], creator presents the basic whole number coefficient computerized channel along Infinite motivation reaction (IIR) structure for Finite drive reaction (FIR), which is band-stop straightforward number coefficient advanced channel. The structured technique used to sift through the gauge float and force recurrence obstruction, and ECG signal without examination.

In [10], creators structured new versatile strategy for denoising the ECG signal. The proposed multistage technique can dispense along numerous sorts of ancient rarities because of various reference inputs so it tends to be applied to the frameworks where different kinds of impedance are discovered, given that the apriori information about the obstruction is accessible.

In [11], creator proposed late strategies for the pattern meander expulsion and electrical cable obstruction decrease in ECG signals utilizing experimental wavelet change (EWT).

In [12], creator proposed another commotion decrease technique that can apply to crude ECG flags all together acquire a higher sign to-clamour proportion (SNR) for additional processing. Creators utilized the wavelet change and dynamic thresholding way to deal along limit specific sorts of clamour installed in crude ECG signals.

Above all the ECG signal filtering methods, for decisionmaking process, it's important to work on QRS complex and ST segment extraction, there are many methods presented for the same in [13]-[23]. The features extraction and classification part of CVD detection frameworks, some of the recent methods reviewed below for ECG based disease detection.

In [24], creator presents the technique for proficient framework for acknowledgment of the premature ventricular constriction from the heart maladies thumps and ordinary pulsates. This framework incorporates three fundamental stages: de-noising module, include extraction module and classifier module.

In [25], ECG signal analysis and characterization strategy utilizing wavelet vitality histogram technique and support vector machine (SVM).They structured the cardiovascular arrhythmia discovery in the ECG signal dependent on three phases including ECG signal pre-processing, highlight extraction and pulses order.

In [26], an effective and simple to-interpret strategy of heart illness order proposed dependent on novel component extraction techniques and correlation of classifiers. Creators described the appropriations by test quintiles which beat test implies. Creator examined the highlights extraction technique utilizing three classifiers utilizing measurement decreased highlights by PCA: stepwise discriminate analysis (SDA), SVM, and LASSO calculated relapse.

In [27], the ongoing work on CVD discovery utilizing ECG signal detailed. Creators utilized sign processing and neural systems procedures for processing ECG signals comprising of extricating highlights from ECG signal so as to distinguish the kinds of CVD's. 
In [28], the dynamical ECG acknowledgment structure proposed for CVD's and human location utilizing the dynamical neural learning system. The proposed technique comprises of two stages: a preparation stage and a test stage. In the preparation stage, cardiovascular elements inside ECG signals is removed (approximated)accurately by utilizing spiral premise work (RBF) neural systems through deterministic learning component. They got heart framework elements is represented and put away in consistent RBF systems.

In [29], late strategy proposed for the QRS Complex location from the ECG signal utilizing 1-D convolutional neural system (CNN). The CNN comprises of article level and part-level CNNs for extricating distinctive grained ECG morphological highlights consequently. All the removed morphological highlights are utilized by multi-layer recognition (MLP) for QRS complex discovery. Also, creator received the ECG signal pre-processing technique which just contains contrast activity in transient area.

In [31], proposed approach is implemented using ML-libs and Scala language on Apache Spark framework. Main challenge in ECG classification was to handle the irregularities in the ECG signals. ECG heartbeats are grouped under three main categories that is normal, PVC, PAC, and other.

From the recent literature analysis, QRS complex and ST segment detection features extraction may leads to the computation complexity problem, the work proposed in this paper is different in many ways as mainly focused on computationally efficient as well as higher accuracy for CVD detection.

\section{Methodology}

This section presents the proposed methodology for CVD detection. Fig. 2 shows the architecture for proposed technique. As observed in figure, the raw ECG signal given as input for CAD system for decision-making process whether it's functioning normal or CVD. In first step, applied the preprocessing algorithm to remove the noises and artefacts without loss of generality along efficiency and robustness in results. The pre-processing ECG signal given input to P, Q, $\mathrm{R}, \mathrm{S} \& \mathrm{~T}$ waves detection utilizing proposed method, the detected waves are used to form the QRS complex and ST segments. QRS complex and ST segments fused to get the final feature vector as both waves the important information of changes in heart behaviour. As the QRS a complex and ST segment both contains large amount information, this may lead the computationally inefficient while applying the classifier for the detection purpose as well as less detection rate. In this work, applied the normalized HOS technique in which first wavelet packet decomposition method applied to get total 90 unique features. Those features further normalized, hence called as NHOS. Five different classifiers are applied to perform the detection in this work.

\section{A. Pre-Processing}

Hybrid filtering method for the ECG signal pre-processing is designed. Algorithm 1 shows the working of proposed preprocessing strategy. The Butter worth channel intended to evacuate the benchmark meandering. Further, second request step channel at $60 \mathrm{~Hz}$ is applied, contingent upon the way that signs originate from European or US accounts to expel powerline obstruction. The use of both filtering methods assures that the artefacts available in raw ECG signal also suppressed. After successful removal of unwanted information from the ECG signal, the process of wave's extraction and features formation are applied.

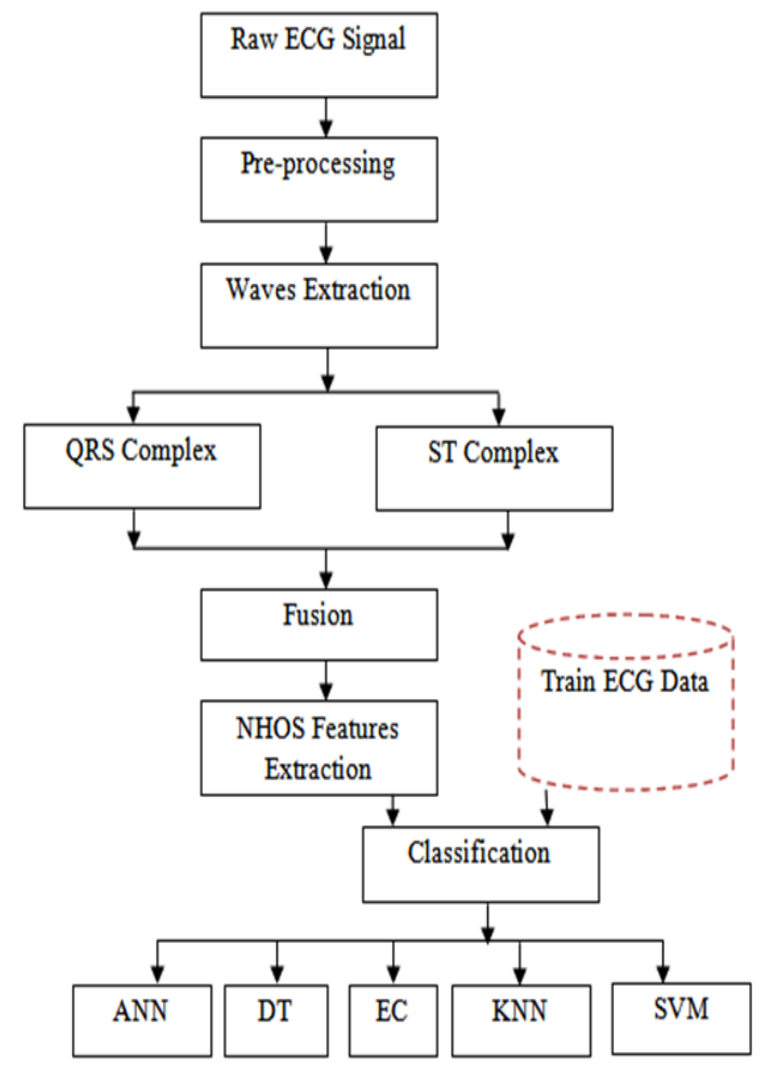

Fig. 2. Proposed CVD Detection System.

\begin{tabular}{|l|}
\hline Algorithm 1: ECG Signal Denoising \\
\hline Inputs \\
$S:$ ECG signal \\
$\delta=2$ : cut off frequency \\
$\sigma=1000:$ data sampled frequency \\
$\varepsilon=50 \mathrm{~Hz}$ \\
Output \\
$P$ : Pre-processed signal \\
$\quad$ 1. Acquire input raw signal $S$ \\
$\quad$ 2. Perform noise insertion explicitly in $S$ \\
$\quad$ 3. Baseline wander removal \\
$B=$ Butterworth (S, $\left(\frac{\delta}{\left(\frac{\sigma}{2}\right)}\right)$, 'high') \\
$\quad$ 4. Design notch filter \\
$P=$ notch $(B, \varepsilon)$ \\
$\quad$ 5. Return $P$ \\
\hline
\end{tabular}




\section{B. Waves Extraction}

The pre-processed ECG signal used for the waves detection and extraction utilizing the proposed thresholding based method. First extract all the waves such as Q, R, S, P, \& $\mathrm{T}$, and then form the QRS complex and ST segment for the features extraction process. Along efficiency of wave's detection and extraction, the main objective is that wave's extraction algorithm should take minimum computation efforts. The technique of thresholding is dynamic in which the threshold value is fixed and computed as per the input ECG signal. This method is start from the signal normalization to QRS complex and ST segment extraction. Algorithm 2 shows the proposed methodology designed in this paper.

As observed in Algorithm 1, the first step is to perform the normalization of pre-processed signal as:

$A=\frac{P^{2}}{\max \left(\left|P^{2}\right|\right)}$

\begin{tabular}{|l}
\hline Algorithm 2: Waves Extraction \\
Inputs \\
$P:$ Pre-processed ECG signal \\
Output \\
QRS \\
$S T \quad$ \\
\hline 1. $\quad$ Compute length $N$ of $P$ \\
2. A: Signal Normalization utilizing Eq. (1) \\
3. AS: Average signalling utilizing Eq. (5) \\
4. $\quad \alpha$ : Mean of Signal AS \\
5. AS: Apply the threshold on AS utilizing Eq. (6) \\
6. $\quad$ [L, R]: Estimate the left \& right waves \\
7. $\quad$ Rwave: Extract $R$ wave utilizing Eq. (7) \\
8. $\quad$ Qwave: Extract $Q$ wave utilizing Eq. (8) \\
9. Swave: Extract $S$ wave utilizing Eq. (9) \\
10. Pwave: Extract $T$ wave utilizing Eq. (10) \\
11. Twave: Extract $P$ wave utilizing Eq. (11) \\
12. QRS: [Qwave, $R$ wave, Swave] \\
13. ST: [Swave, Twave] \\
14. Return (QRS, ST) \\
15. Stop \\
\hline
\end{tabular}

Where, $\mathrm{P}$ is the pre-processing ECG signal. The normalized signal further used for the computation of average signalling utilizing the convolution operations as:

$B=\frac{\text { Ones }(1,31)}{31}$

The temporary array of matrix 1 's of size $1 * 31$ which is used to perform the convolution along normalized signal $A$ as:

$\mathrm{C}=\operatorname{conv}(A, B)$

$\mathrm{C}=C(15+[1: N])$
The convolution output used to compute the average signal as:

$A S=\frac{\mathrm{C}}{\max (|\mathrm{C}|)}$

The normalization and average signalling of original preprocessed signal helps to minimize the overhead of computation burden while estimating the waves, also improves the accuracy of waves extraction. The dynamic threshold value is computed by taking the mean of $A S$ signal. The computed threshold value applied to select the waves those satisfies the threshold value as:

$A S=\mathrm{AS}>\alpha$

The left and right waves computed utilizing Differences and Approximate Derivatives (DAD) operator. The DAD computes the differences between adjacent elements of AS along the first array dimension whose size does not equal. This returns the signals of matrix starting from -1 (left) to 1 (right). Thus, got the left wave and right wave for the signal. From the left and right waves perform the subtract delay operations to suppress the low-pass and high-pass filtering. After the localization of left and right waves, extract the $P, Q$, $R, S$, \& $T$ waves as utilizing the minimum and maximum operations on $L$ (left) \& $R$ (right) waves detected.

Rwave $=\max (\mathrm{P}(\mathrm{L}: \mathrm{R}))$

Qwave $=\min (\mathrm{P}(\mathrm{L}:$ Rwave $))$

Swave $=\min (\mathrm{P}(\mathrm{L}: \mathrm{R}))$

Pwave $=\max (\mathrm{P}(\mathrm{L}:$ Qwave $\mathrm{QT}))$

Twave $=\max (\mathrm{P}($ Swave: $R))$

The QRS complex and ST segments formed utilizing the extracted waves.

\section{Features Extraction}

As the QRS complex and ST segments are having big number of data which may be the inefficient in terms of accuracy as well as computational efforts while applying the machine learning technique for CVD detection, here introduced the higher order statistics (HOS) based features extraction technique [30]. First extract the HOS features utilizing the $4^{\text {th }}$ level wavelet packet decomposition technique, followed by the operator's kurtosis and skewness.

The first and second order statistics not enough to improve the detection accuracy, used the HOS that computes the higher order moments $(m 2, m 3, m 4)$ and non-linear combinations of higher order moments which are known as cumulates (c2, c3, c4). The cumulates are of second (c2), third (c3), and forth (c4) order statics of each wavelet packet decomposition coefficient (at each level). Applied this method on QRS+ST matrix which extracts the 90 unique and complex features. The features complex, hence here add the normalization step to HOS outcomes, called as NHOS features. Then perform the normalization of features utilizing the Eq. (1). The NHOS features are used for the classification purpose. 


\section{Classification}

Here five different machine learning techniques for the classification process are used in which the NHOS features of input raw ECG signal consider as test input to detect whether it has CVD or not. The classifiers such as Artificial Neural Network (ANN), k-nearest neighbors (KNN), Support Vector Machine (SVM), Ensemble Classifier (EC), Decision Tree (DT) used to investigate performance of proposed method. For classifiers like SVM, EC, DT, and KNN used the 10-fold cross validation technique, whereas the ANN used the ratio $70 \%, 15 \%, 15 \%$ ratio of training, validation, and testing, respectively.

\section{RESULT ANALYSIS}

MATLAB is used for CVD detection and PhysikalischTechnische Bundesanstalt (PTB) ECG dataset [32] is used for the experiment. For both heart disease patients and healthy patients ECG signals were recorded by the Professor Michael Oeff, M.D. The 545 ECG signals were collected for different subjects consisting of both CVD and normal. There are total 468 ECG signals affected by CVD and 77 samples are normal.

Here the training is performed on all 545 ECG samples, and then performed the classifications by utilizing five different classifiers. First demonstrate the outcomes of preprocessing algorithm, and then present the results related to accuracy.

Fig. 3 shows the crude ECG signal which is misshaped because of the presence of floats, benchmark meanders, and extra clamor because of the ongoing condition catching procedures. Further the sign ECG is pre-handled utilizing the half and half methodology wherein disentangle the procedure of ECG signal denoising by utilizing the two understand channels at tentatively confirmed setups. Fig. 4 shows the result denoising which shows the reasonable distinction between unique ECG signals and denoised ECG signal. In following graphs, $\mathrm{X}$ axis presents milliseconds and $\mathrm{Y}$ axis presents millivolts.

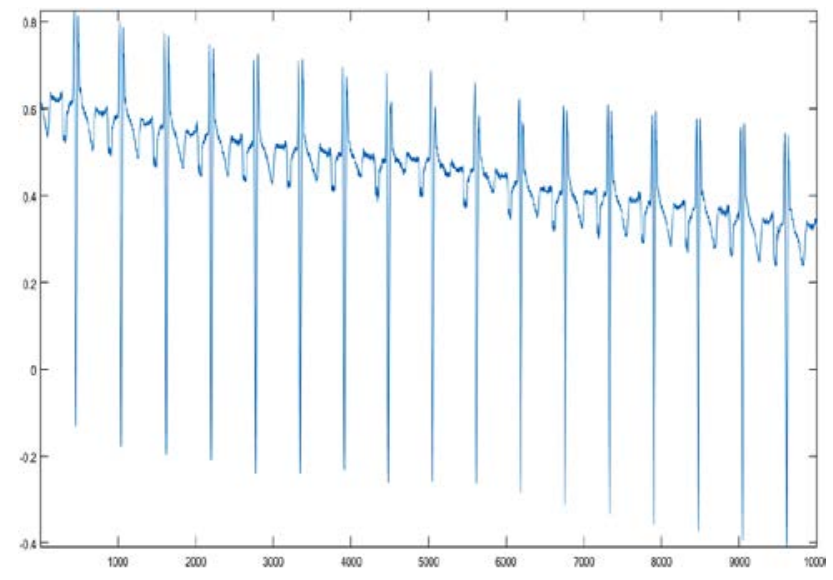

Fig. 3. Original ECG Signal.

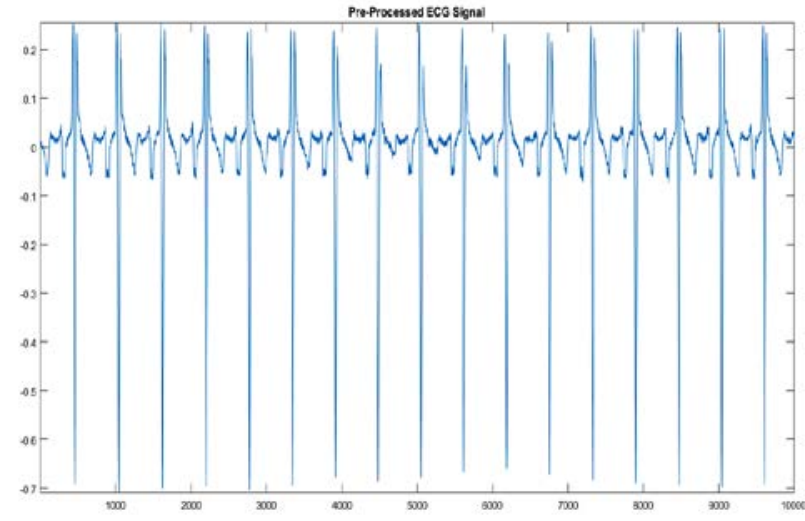

Fig. 4. Pre-Processed ECG Signal utilizing Hybrid Filtering.

\section{DISCUSSION}

Table I shows the performances in terms of Mean Square Error (MSE), Signal to Noise Ratio (SNR), and time complexity. The average performance is measured for 50 ECG signals from the given dataset. The time complexity is shows nothing more difference in time complexity performance. Suggested way is effective and efficient for preprocessing of ECG signal.

This paper present the performance investigation of proposed method called QRS_ST + NHOS utilizing five different classifiers. The proposed model is compared along QRS_ST (without utilizing any features extraction technique), and QRS_ST+HOS (without normalization) to claim the efficiency. Table II shows the detection accuracy performance in terms of for all classifiers.

As observed in Tables II to IV, the performance of proposed QRS_ST + NHOS shows the significant improvement in accuracy, precision, and recall rates compared to the QRS_ST and QRS_ST+HOS. In an average the ANN classifier shows the best performance as compared to other classifiers. The DT classifier shows the worst performance among other classifiers. The results of QRS_ST demonstrates that, using only QRS and ST segments, the classification results is not improved, but by using the said features extraction technique the performance is enhanced. Similarly, the using NHOS demonstrates the benefits of applying the normalization technique on extracted features. Table $\mathrm{V}$ shows the average computation time required for CVD detection and classification time (using KNN classifier). As the size of QRS_ST is larger, it takes long time for the classifier to perform the features matching, hence takes more time for the detection as compared to QRS_ST+HOS and QRS_ST+NHOS. There is negligible difference between both HOS based model and NHOS based model, as NHOS mainly introduced to improve the accuracy rate.

Finally, the accuracy comparison along similar works presented in Table VI. The results show the performance improvement utilizing proposed model over the methods presented recently. 
TABLE I. PRE-Processing AlgORITHM ANALYSIS

\begin{tabular}{|l|l|l|l|}
\hline Methods & MSE & SNR & $\begin{array}{l}\text { Time Complexity } \\
\text { (seconds) }\end{array}$ \\
\hline Butterworth & 0.3545 & 10.55 & 0.148 \\
\hline Notch & 0.3323 & 10.78 & 0.112 \\
\hline Adaptive Filtering & 0.2566 & 11.89 & 0.193 \\
\hline Hybrid & 0.1973 & 13.33 & 0.156 \\
\hline
\end{tabular}

TABLE II. CVD DETECTION ACCURACY (\%) ANALYSIS

\begin{tabular}{|l|l|c|l|}
\hline & QRS_ST & QRS_ST+HOS & QRS_ST+NHOS \\
\hline ANN & 89.77 & 91.98 & 94.47 \\
\hline DT & 80.47 & 84.11 & 86.82 \\
\hline EC & 83.29 & 88.35 & 91.76 \\
\hline KNN & 82.58 & 90.23 & 92.23 \\
\hline SVM & 76.94 & 89.76 & 91.76 \\
\hline
\end{tabular}

TABLE III. CVD DETECTION PRECISION (\%) ANALYSIS

\begin{tabular}{|l|l|l|l|}
\hline & QRS_ST & QRS_ST+HOS & QRS_ST+NHOS \\
\hline ANN & 89.77 & 91.98 & 94.47 \\
\hline DT & 88.66 & 84.11 & 86.82 \\
\hline EC & 84.17 & 88.35 & 91.76 \\
\hline KNN & 85.42 & 90.23 & 92.23 \\
\hline SVM & 85.24 & 89.76 & 91.76 \\
\hline
\end{tabular}

TABLE IV. CVD DETECTION RECALL (\%) ANALYSIS

\begin{tabular}{|l|l|l|l|}
\hline & QRS_ST & QRS_ST+HOS & QRS_ST+NHOS \\
\hline ANN & 89.77 & 91.98 & 95.47 \\
\hline DT & 65.93 & 75.38 & 74.65 \\
\hline EC & 97.01 & 99.33 & 91.65 \\
\hline KNN & 88.01 & 78.67 & 78.67 \\
\hline SVM & 89.29 & 100 & 100 \\
\hline
\end{tabular}

TABLE V. COMPUTATION EFFORTS ANALYSIS (SECONDS)

\begin{tabular}{|l|l|l|l|}
\hline & QRS_ST & QRS_ST+HOS & QRS_ST+NHOS \\
\hline CVD Detection & 3.79 & 3.25 & 3.23 \\
\hline Classification time & 1.89 & 1.45 & 1.42 \\
\hline
\end{tabular}

TABLE VI. ACCURACy RATE EVALUATION ALONG SimiLAR WORKS

\begin{tabular}{|l|l|}
\hline & Accuracy (\%) \\
\hline$[26]$ & 88.52 \\
\hline$[27]$ & 82.5 \\
\hline$[29]$ & 92.5 \\
\hline Proposed & 94.47 \\
\hline
\end{tabular}

\section{CONCLUSION AND FUTURE WORK}

The aim of this paper is to present the methodology for the CVD detection utilizing the ECG signals. The proposed framework is consisting of pre-processing, wave's extraction, features extraction and classification. At first step, paper proposed the hybrid filtering technique to filter out different type's noises and artefacts. The proposed hybrid filtering technique is simulated and evaluated along existing filtering techniques in terms of MSE, SNR and Time complexity parameters. For wave's extraction, the lightweight and efficient dynamic threshold based algorithm are proposed. Here first locate the left and right waves, and then extract the Q, R, S, T, \& P waves. Utilizing the extracted waves, QRS complex and ST segments extracted. To minimize the computational efforts and improve the accuracy, here applied the NHOS features extraction technique on extracted QRS complex and ST segments. In classification step, five different classifiers applied and investigate their performances in terms of accuracy, precision, recall, and computational time. The proposed method shows the significant improvement over the recent methods. For future work, use of different ECG heart datasets are suggested.

\section{REFERENCES}

[1] Tu Ya, Zhou Runjing, Zhang Fei, "ECG Signal Preprocessing Based on Change Step Iteration of the LMS Adaptive Filtering Algorithm", 2009 World Congress on Computer Science \& Information Engineering.

[2] D. Balasubramaniam, D. Nedumaran, "Implementation of ECG Signal Processing \& Analysis Techniques in Digital Signal Processor based System", MeMeA 2009 - International Workshop on Medical Measurements \& Applications, Cetraro, Italy, May 29-30, 2009.

[3] Ioan Tudosa, Narcis Iulian Adochiei, \& Razvan Ciobotariu, "New Aspects in ECG Signal Processing Utilizing Adaptive Filters", 7th International Symposium On Advanced Topics in Electrical Engineering (ATEE), 2011.

[4] Lukas Smolarik, Adriana Libosvarova, Dusan Mudroncik, Peter Schreiber, "Non-contact ECG Signal Processing", 6th IEEE International Conference Intelligent Systems, 2012.

[5] Anil Chacko \& Samit Ari, "Denoising of ECG Signals utilizing Empirical Mode Decomposition Based Technique”, IEEE International Conference on Advances in Engineering, Science \& Management (ICAESM), pp. 6-9, 2012.

[6] Mehmet Ustundag, Muammer Gokbulut, Abdulkadir Sengur \& Fikret Ata, "Denoising of Weak ECG Signals by utilizing Wavelet Analysis \& Fuzzy Thresholding”, Springer Network Modeling Analysis in Health Informatics \& Bioinformatics , Volume 1, Issue 4, pp 135-140, 2012.

[7] Lukas Smital, Martin Vitek, Jiri Kozumplik, \& Ivo Provaznik, "Adaptive Wavelet Wiener Filtering of ECG Signals", IEEE Transactions On Biomedical Engineering, Volume 60, Issue 2, pp. 437 445, 2013.

[8] Mohammed Assam Ouali \& Kheireddine Chafaa, "SVD-Based Method for ECG Denoising", IEEE International Conference on Computer Applications Technology (ICCAT), pp. 1 - 4, 2013.

[9] Dong Jingwei, Jiang Wenwen, "Design of Digital Filter on ECG Signal Processing", Fifth International Conference on Instrumentation \& Measurement, Computer, Communication \& Control, 2015.

[10] Rizwan Qureshi, Muhammad Uzair, Khurram Khurshid, "Multistage Adaptive Filter for ECG Signal Processing", International Conference on Communication, Computing \& Digital Systems (C-CODE), 2017.

[11] Omkar Singh, Ramesh Kumar Sunkaria, "ECG signal denoising via empirical wavelet transform", Australas Phys Eng Sci Med, 2017.

[12] Pandit D., Zhang L., Liu C., Aslam N., Chattopadhyay S., Lim C.P. "Noise Reduction in ECG Signals Utilizing Wavelet Transform \& Dynamic Thresholding", In: Bhatti A., Lee K., Garmestani H., Lim C. (eds) Emerging Trends in Neuro Engineering \& Neural Computation. Series in BioEngineering. Springer, Singapore, 2017.

[13] Arzeno NM, Deng ZD, Poon CS, "Analysis of first-derivative based QRS detection algorithms", IEEE Trans Biomed Eng. 2008;55:478-484.

[14] Keselbrener L, Keselbrener M, Akselrod S, "Nonlinear high pass filter for R-wave detection in ECG signal", Med Eng Phys. 1997;19:481-484. 
[15] Sharma T, Sharma KK, "QRS complex detection in ECG signals utilizing locally adaptive weighted total variation denoising", Comput Biol Med. 2017;87:187-199.

[16] Trahanias PE, "An approach to QRS complex detection utilizing mathematical morphology", IEEE Trans Biomed Eng. 1993;40:201-205.

[17] Coast DA, Stern RM, Cano GG, Briller SA, "An approach to cardiac arrhythmia analysis utilizing hidden Markov models", IEEE Trans Biomed Eng. 1990;37:826-836.

[18] Zidelmal Z, Amirou A, Adnane M, Belouchrani A, "QRS detection based on wavelet coefficients", Comput Methods Programs Biomed. 2012;107:490-496.

[19] Hamdi S, Ben AA, Bedoui MH, "Real time QRS complex detection utilizing DFA \& regular grammar", Biomed Eng Online. 2017;16:31.

[20] Phukpattaranont P., "QRS detection algorithm based on the quadratic filter", Expert Syst Appl. 2015;42:4867-4877.

[21] Farashi S., "A multiresolution time-dependent entropy method for QRS complex detection", Biomed Signal Process Control. 2016;24:63-71.

[22] Zhou Y, Hu X, Tang Z, Ahn AC., "Sparse representation-based ECG signal enhancement \& QRS detection", Physiol Meas. 2016;37:2093.

[23] Jung WH, Lee SG., "An R-peak detection method that uses an SVD filter \& a search back system", Comput Methods Programs Biomed. 2012;108:1121-1132.

[24] Ataollah Ebrahim Zadeh, Ali Khazaee, Vahid Ranaee, "Classification of the electrocardiogram signals utilizing supervised classifiers \& efficient features", computer methods \& programs in biomedicine vol. 99, pp.179-194, 2010.
[25] Prof. Alka S. Barhatte, Dr. Rajesh Ghongade, Abhishek S. Thakare, "QRS Complex Detection \& Arrhythmia Classification utilizing SVM", 2015 International Conference on Communication, Control \& Intelligent Systems (CCIS).

[26] Huang, Rong \& Yingchun Zhou. "Disease Classification \& Biomarker Discovery Utilizing ECG Data” BioMed research international vol. 2015 (2015): 680381.

[27] Shalin Savalia, Eder Acosta, Vahid Emamian, "Classification of Cardiovascular Disease Utilizing Feature Extraction \& Artificial Neural Networks", Journal of Biosciences \& Medicines, vol. 5, pp. 64-79, 2017.

[28] Muqing Deng a, Cong Wangb, Min Tang c, Tongjia Zheng, "Extracting cardiac dynamics within ECG signal for human identification \& cardiovascular diseases classification", Neural Networks vol. 100, pp. 70-83, 2018.

[29] Xiang, Yande et al. “Automatic QRS complex detection utilizing twolevel convolutional neural network” Biomedical engineering online vol. 17,1 13. 29 Jan. 2018.

[30] Yakup Kutlu, Damla Kuntalp, "Feature extraction for ECG heartbeats utilizing higher order statistics of WPD coefficients", Comput Methods Programs Biomed, vol. 105, no. 3, pp.57-67, 2012.

[31] Fajr Ibrahem Alarsan, Mamoon Younes “Analysis and classification of diseases using heartbeat features and machine learning algorithms” Springer open, journal of Big data, pp 1-15, 2019.

[32] https://www.physionet.org/physiobank/database/ptbdb/. 